M. Petschler ${ }^{1}$ M. Stiller ${ }^{1}$ B. Hoffmeister ${ }^{1} \cdot$ R. Witkowski ${ }^{2}$ C. Opitz ${ }^{3}$ J.S. Bill ${ }^{4} \cdot$ H. Peters ${ }^{2}$

${ }^{1}$ Abteilung für Zahnärztliche Chirurgie und Röntgenologie, Klinik für Kieferchirugie und plastische Gesichtschirurgie, Universitätsklinikum Benjamin Franklin, Berlin

${ }^{2}$ Institut für Medizinische Genetik, Universitätsklinikum Campus Mitte, Humbold-Universität, Berlin

${ }^{3}$ Abteilung für Kieferorthopädie, Universitätsklinikum Campus Mitte, Humbold-Universität, Berlin

${ }^{4}$ Kliniken und Polikliniken für Mund-, Kiefer- und Gesichtschirurgie,

Bayerische Julius-Maximilians-Universität, Würzburg

\title{
Klinische und molekulargenetische Befunde bei Familien mit Cherubismus über 3 Generationen
}

\section{Clinical and molecular genetic observations on families with cherubism over three generations}

\section{Mund Kiefer GesichtsChir (2003) DOI 10.1007/s10006-002-0444-x}

Im Abschnitt „Material und Methode“ stand irrtümlich:

In der Familie A fanden sich 3 (Abb. 1, Stammbaum Familie A), in der Familie B insgesamt 10 sichere Merkmalsträger in 3 Generationen (Abb. 2, Stammbaum Familie B).

Richtig ist:

In der Familie A fanden sich 3, in der Familie B insgesamt 10 sichere Merkmalsträger in 3 Generationen (Abb. 1, Stammbaum Familie B).

\section{Petschler}

Abteilung für Zahnärztliche Chirurgie und Röntgenologie, Klinik für Kieferchirugie und plastische Gesichtschirurgie, Universitätsklinikum Benjamin Franklin, Aßmannshauser Straße 4-6, 14197 Berlin, Tel.: 030-84456112, Fax: 030-84456115, E-mail: michael.petschler@ukbf.fu-berlin.de 